\title{
Integrated Software Development for Embedded Robotic Systems
}

\author{
Sebastian Wätzoldt, Stefan Neumann, Falk Benke, and Holger Giese \\ [sebastian.waetzoldt|stefan.neumann|holger.giese]@hpi.uni-potsdam.de \\ falk. benke@student.hpi.uni-potsdam.de \\ Hasso Plattner Institute for Software Systems Engineering \\ University of Potsdam, Germany
}

\begin{abstract}
In the recent years, improvements in robotic hardware have not been matched by advancements in robotic software and the gap between those two areas has been widening. To cope with the increasing complexity of novel robotic embedded systems an integrated and continuous software development process is required supporting different development activities and stages being integrated into an overall development methodology, supported by libraries, elaborated tools and toolchains. For an efficient development of robotic systems a seamless integration between different activities and stages is required. In the domain of automotive systems, such an overall development methodology, consisting of different development activities/stages and supported by elaborated libraries, tools and toolchains, already exists. In this paper, we show how to adapt an existing methodology for the development of automotive embedded systems for being applicable on robotic systems.
\end{abstract}

\section{Introduction}

In novel robotics applications steady improvements in robotic hardware is not matched by advancement in robotic software leading to an increasing gap between those two areas. The increasing complexity of modern robotic systems requires to further support several different software development activities such as modeling, simulation and testing that allow the incremental development of robot systems, starting with a single sensor and resulting in a complex application. Elaborated tools and toolchains are required to support the different activities and integrate them into an overall and well structured development methodology. To realize an efficient software development process, on the one hand, one has to provide libraries supporting individual development activities at different levels, e.g., at the level of individual sensors and control functions or at the level of systems or sub-systems, being incrementally composed. On the other hand, a seamless migration between individual development activities and stages has to be achieved. Furthermore, one crucial aspect that needs to be considered for a large portion of robotic systems is real-time behavior.

Accordingly, the following aspects need to be considered for bridging the gap between hardware and software development in novel robotic systems: (I) An 
overall methodology is required that supports (II) different development activities like modeling, simulation and testing at (III) different stages, e.g., simulation, prototyping and (pre-)production. Such a methodology has to be supported by (IV) elaborated tools and (V) libraries integrated into (VI) an overall toolchain allowing a seamless migration between the different development stages and artifacts. (VII) Simulation and testing support is required for the stages, allowing to validate created functionality, developed sub-systems or systems, e.g., by providing executable functional models, simulation environments and plant models. (VIII) Last but not least, real-time constraints need to be reflected.

As an example, in the automotive domain large complex real-time embedded systems are developed using different development stages, e.g., simulation, prototyping, and pre-production. Advanced tools and libraries have emerged during the recent years, integrated into sophisticated toolchains supporting different development stages as well as a seamless migration between them. To deal with the increasing complexity and to further reduce software development costs as well as time, advanced frameworks for the distributed and component-based development have been developed. In this paper, we propose adapting the existing software development methodology used in the domain of automotive embedded systems to support the software development of novel, complex embedded robotic systems. The proposed methodology includes an overall development process consisting of tools included into an overall toolchain as well as libraries. We apply this existing approach to the domain of robotic systems and evaluate as a proof of concept, which modifications have to be made. The approach is evaluated using a mobile robot developed according to the adapted methodology. Special attention is given to real-time constraints that need to be considered in a slightly different way than in the case of automotive real-time embedded systems. Therefore, we show a new approach for combining hard and soft real-time behavior in the existing automotive framework.

The remainder of this paper is organized as follows. Section 2 briefly discusses the foundations of robotic as well as automotive systems and introduces a running example for this paper. Section 3 describes our development approach including different stages and highlights our used tools as well as simulation and verification possibilities. The paper discusses related work in Section 4 and concludes in Section 5

\section{Foundations - Robotic and Automotive Systems}

\subsection{Robot Laboratory}

For the evaluation of our research activities, we use our MDELab robot laboratory consisting of three Robotino robots ${ }^{1}$ The robots can be equipped with several sensors (e.g., laser scanner, infrared (IR) distance sensors, GPS like indoor navigation systems) as well as different actuators (e.g., servo motors, omnidirectional drive, gripper). The general idea of our evaluation scenario is the

Ww. festo-didactic.com 
realization of a variable production setting, where robots are capable of transporting small pucks (representing goods in a production system) to different locations. Robots have to fulfill different requirement, e.g., they have to provide basic functionality like moving and avoiding obstacles in hard real-time (reacting on obstacles within a few milliseconds). Further, the robots have to reflect high level goals, e.g., energy saving of the battery, short routing to the destination points and optimizing the throughput while transporting the pucks. While basic functionalities, such as obstacle avoidance, have to be realized in hard real-time, we use existing libraries to realize higher functionalities such as path planning or creating a map by evaluating measured distance values. The latter can rarely be realized under hard real-time constraints because of insufficient libraries Furthermore, we run a RTAI Linux operating system ${ }^{3}$ on the robot to enable hard real-time execution.

As a running example, we use a single robot with the following hardware/ software configuration: The robot has three wheels realizing an omnidirectional drive. The drive unit provides an incremental encoder to realize odometry functionality, which calculates the relative position over time according to the drive speed and the orientation of the omnidirectional drive of the robot. Due to the fact that this odometry calculation becomes more and more imprecise over time, we use an additional GPS like (NorthStar ${ }^{4}$ indoor navigation system to correct the position in the long run. IR distance sensors are used to avoid obstacles during movement. A more complex navigation logic uses these sensors for maintaining a map ${ }^{2}$ as well as computing an appropriate route for the robot while avoiding obstacles.

\subsection{Automotive Development Process}

A commonly applied development process for the development of automotive embedded real-time systems according to [4] is depicted on the left in Fig. 2. The development process includes three different stages, namely the simulation, prototyping and pre-production stage. During the simulation stages models are extensively used for realizing control functionality as well as for representing the environment. At the prototyping stage, a transition from a model-based to a software centric development approach is realized. Often, this is achieved by using code generators that automatically derive source code from the models used in the previous stage. In the pre-production stage, more and more aspects of the real system are involved, e.g., by using prototyping HW including the processor type (with additional debugging support) that is later used. Furthermore, parts of the real plant enable a realistic validation of the real-time behavior.

\footnotetext{
${ }^{2}$ For path planning and creating a map the MRPT library is used (www.mrpt.org).

www.rtai.org

WW. evolution.com/products/northstar/
} 


\subsection{AUTOSAR}

The AUTomotive Open System ARchitecture was invented to further support the development of complex and distributed systems. AUTOSAR ${ }^{5}$ is the new de facto standard in the automotive domain. It defines a layered architecture, standardized communication mechanism and a whole development methodology. Furthermore, it supports the interaction between different car manufactures and suppliers. Figure 1 gives an overview of the layered AUTOSAR architecture. The layer at the bottom represents the real hardware including microcontroller and communication busses. An abstraction layer on top of the real hardware, included in the basic software layer, offers standardized interfaces for accessing the HW. Further functionality realizing the OS behavior as well as functionality for realizing communication is included in the basic software layer. The AUTOSAR runtime environment (RTE) is responsible for realizing the communication from and to the top soft-

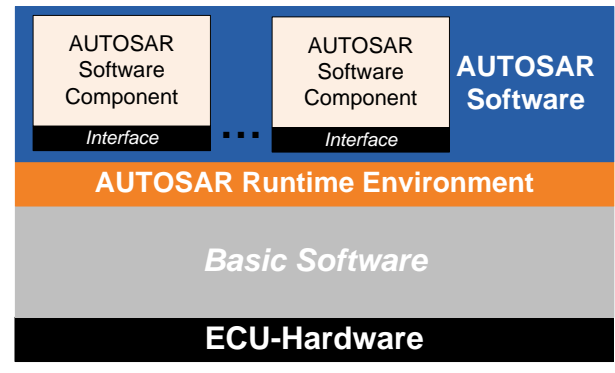

Fig. 1. The layered AUTOSAR architecture according to the specification in 12 . ware application layer. Software components (SWCs) realize application

functionality at the layer on top. There, the architecture style changes from a layered to a component based approach 12 . SWCs communicate over welldefined ports using AUTOSAR interfaces, which are realized by the RTE layer. Each SWC consists of an arbitrary number of so-called Runnables that specify the behavior entities of each component ${ }^{6}$ Such Runnable entities are mapped on OS tasks, which are scheduled and handled by the operation system included in the basic software layer.

\subsection{Automotive vs. Robotic Systems}

In an automotive embedded system, usually applications are developed in such a fashion that hard real-time capable functionalities are separated from soft real-time applications. For example, it is quite common to deploy soft and hard real-time functionality on disjoint execution nodes and direct communication between them is avoided.

For robotic systems it is quite common to combine soft and hard real-time behavior into one application. For example, a mobile robot needs to avoid obstacles under hard real-time during navigation while calculating a route and updating a map. Both functionalities need to be combined while predicting the

\footnotetext{
$\sqrt[5]{\text { www . autosar .org }}$

${ }^{6}$ The functionality of a Runnable can be realized by a $\mathrm{C} / \mathrm{C}++$ function.
} 
execution time, e.g., of a route planing algorithm, is often not possible 7 Thus, one difference between automotive and robotic systems concerning the real-time behavior is, that soft and hard real-time capable functionalities need to be more closely linked in robotic systems.

\section{Development Environment}

In this section, we describe our development environment, the tools and libraries used in the different development stages as well as our test and verification possibilities during system development. According to 4], we distinguish three development stages at different levels of abstraction targeting specific key aspects, namely simulation, prototyping and pre-production. Validation and verification activities are applied in each stage according to the given abstraction level. On the left in Figure 2, the overall process including the different stages is shown. In the following, we describe the applied validation and verification activities of each stage in the form of the libraries, methods and tools used. Furthermore, we show how to achieve an AUTOSAR conform system realizing the complex behavior of the robot incrementally developed, validated and verified during the different development stages.

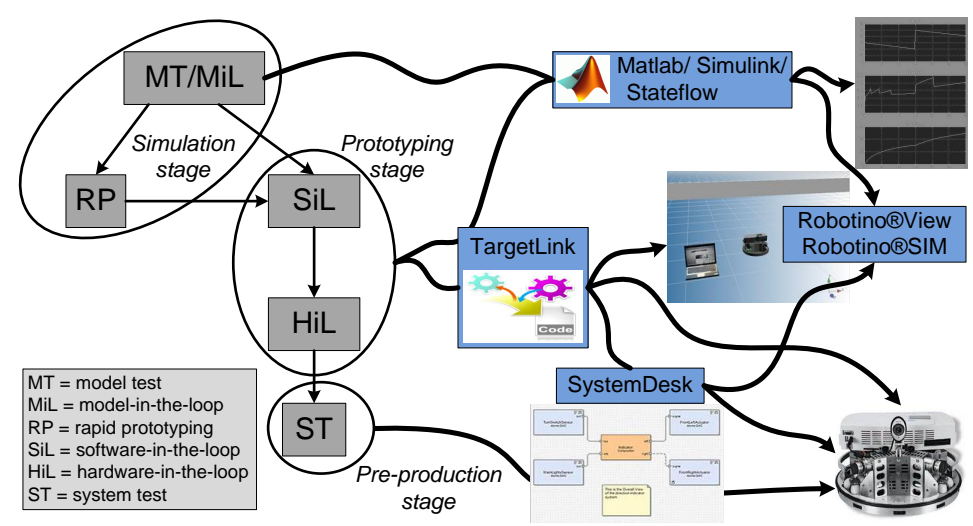

Fig. 2. On the left are the three development stages according to 4 ] in combination with our toolchain during software and system development on the right.

\footnotetext{
${ }^{7}$ Execution time depends on the size of the map, which is usually not known before runtime.
} 


\subsection{Simulation Stage}

Individual functions as well as composed behavior, resulting from multiple individual functionalities, are the subject of the simulation stage. Data flow models in the form of block diagrams (e.g., MATLAB/Simulink) usually in combination with control flow models like Statecharts (e.g. Stateflow) are used 6]. Normally, function development is done independent from platform specific limitations (memory capacity, floating point calculation or effects resulting from discretization). Additionally, environment specific signals and other real sensor values (e.g. produced by $\mathrm{A} / \mathrm{D}, \mathrm{D} / \mathrm{A}$ converter or specific communication messages) are ignored for the sake of sim-

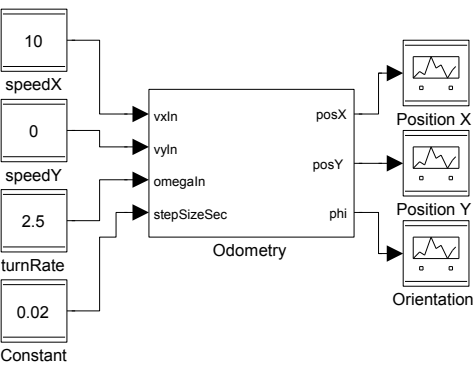

Fig. 3. Odometry in MATLAB, which calculates the position from fix drive speed and turn rate. plicity. The goal of the simulation stage is to prove that the functional behavior can work and as a result provides a first proof of concept for control algorithms.

As depicted in Fig. 2 and according to the aspect (IV), we mainly use the MATLAB tool suite including the Simulink and Stateflow extension in this development stage. Let us consider the MATLAB model shown in Fig. 3, as an example modeling the functionality of an odometry. It reads data from moving sensors to calculate changes in the position over time according the actual orientation and movement speed of the robot. In the simulation stage, such a model is used to apply a so-called model test $(M T)$, where individual functionalities can be simulated sending static input values to the model (e.g., drive speed and turn rate of the robot as in Fig. 3) and plotting the computed output values as shown in Fig. 4. These one-shot/ one-way simulations are typical for the MT step and do not consider the interaction with the environment or a plant model. More complex behavior is constructed and validated in the form of individual functionalities and running model-in-the-loop (MiL) simulations [4] including preliminary environment models of the plant. At this point in time, feedback simulations validate the developed functionality considering the dynamic behavior of the environment. Outputs are sent to the plant model, which itself gives feedback used as input for the function blocks in the next iteration of the MiL simulation. In such a manner, the overall control law can be validated concerning basic constraints like stability, safety or reliability of the system (VII).

In the case of robotic systems, such a plant model can be represented at different levels, e.g., by using models representing a single sensor, the behavior of a single robot using multiple sensors or in the case of a complex simulation realizing the behavior of multiple robots as well as relevant parts of the logical and/or physical environment. Using such a plant model in the context of a MiL simulation, we must bridge the gap between our MATLAB models and the provided model of the plant (VI). For this purpose, on the one hand, we 
use the RobotinoSim simulator in combination with the graphical Robotino View environment ${ }^{8}$ to create plant models (cf. the upper path from the simulation stage in Fig. 2). Therefore, we implemented a block library for MATLAB in our development environment, which allows access to sensors (e.g., distance sensors, bumper, incremental encoder, electrical motors) and actuators according to requirement $(\mathrm{V})$. The sensors and actuators can be accessed individually inside a MiL simulation supporting the validation of the models (VII). The RobotinoSim simulator provides optimal sensor values excluding effects such as sensor noise. Therefore, on the other hand, we can access the HW of the robot directly via a wireless LAN connection. Due to the fact that we use the concrete HW in this simulation setting, we could verify our functionalities and control algorithm with real sensor values including measure errors and sensor noise.

To sum it up, the first column in Table 1 gives an overview of the three tools used and our testing capabilities in the simulation stage as described previously. Additionally on the right in of Fig. 2, one can follow the toolchain used via the flow arrows $9^{9}$ However, we are not limited to the RobotinoSim tool in our development approach. We use this tool to show the proof of concept, but in general it is possible to create block libraries in MATLAB or use existing ones for other robots, simulation frameworks or individual sensors/ actuators.

Table 1. Tools for testing and verification for each development stage.

\begin{tabular}{|c|c|c|c|}
\hline Tool Stage & Simulation & Prototyping & Pre-production \\
\hline MATLAB/Simulink/Stateflow & 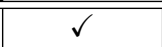 & 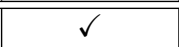 & \\
\hline TargetLink & & $\checkmark$ & \\
\hline SystemDesk & & $\checkmark$ & $\checkmark$ \\
\hline RobotinoSim/View & $\checkmark$ & $\checkmark$ & \\
\hline SystemDesk Simulator & & $\checkmark$ & $\checkmark$ \\
\hline Robotino Robot & $\checkmark$ & $\checkmark$ & $\checkmark$ \\
\hline
\end{tabular}

\subsection{Prototyping Stage}

The focus of this stage changes from design to implementation. While in the simulation stage models are the main artifacts, in this stage the source code plays a major role. In the following, we show how to support the prototyping stage at the level of more isolated functional parts as well as at the level of the system behavior by using the professional, commonly used tools of the automotive domain.

\footnotetext{
${ }^{8}$ In the following, we only mention the simulator, but we always use both tools together in combination. Tools see: www.festo-didactic.com

${ }^{9}$ The described RP flow to the real robot is not shown in the figure.

${ }^{10}$ For example this toolbox: http://petercorke.com/Robotics_Toolbox.html
} 
Function Level - TargetLink: In the automotive domain, code generators are commonly used to derive an implementation for the specific target platform. Usually, the models from the simulation stage are directly used or refined until a code generation step is possible. In our development environment, the tool TargetLink from dSPACE is fully integrated into MATLAB and can automatically derive the implementation from behavior models in form of C-Code. In this step, we use the same MATLAB blocks as discusses in Section 3.1. So, we are able to seamlessly migrate (VI) our functions and control algorithm from the model level, realizing continuous behavior, to the implementation level, realizing a discrete approximation of the original continuous behavior ${ }^{11}$ We can configure several characteristics of the desired target platform/ HW.

Software-in-the-loop (SiL) simulation is a first step from the pure model execution to a code-based testing. Certain assumptions can be validated by replacing more and more models with code. While still executing the software on a host pc and not on the real HW, different effects can be analyzed, which result from chosen configuration
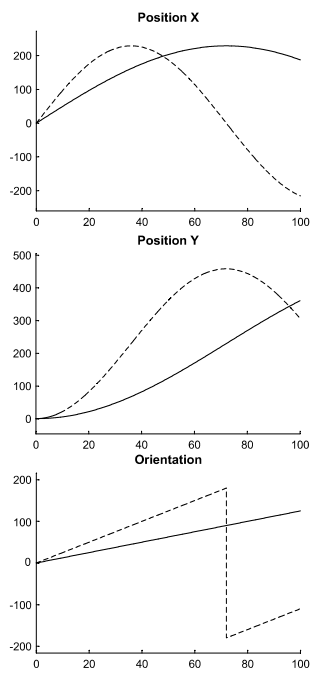

Fig. 4. MiL (dashed line) and SiL simulation values of the odometry block. parameters during code generation. Just as in the MiL simulation case, a SiL simulation can be applied in MATLAB using the generated source code instead of the original model. The developer can switch between the MiL and SiL simulation mode in MATLAB. Therefore, he can easily compare the simulation results. Fig. 4 , for example shows the monitored results of the position as well as the orientation from the MiL and SiL simulation runs of the odometry. The simulations run against the RobotinoSim simulator. In the MiL run (dashed line), appropriate values for the actual position and orientation are calculated. Because of rounding (discretization) effects in the SiL run, the calculated values are much too low. So, the difference between pure model simulation and code generation becomes visible.

The problem in this special example could be fixed by choosing different values for the discretization over time. Calculating the position each 0.02 time units (corresponds to a scheduling with a period of $20 \mathrm{~ms}$, cf. the constant value in Fig. 3 leads to very small offsets in the position, which is often rounded to zero due to discretization. After we identified the problem, we could easily fix it in the model. Instead of a $20 \mathrm{~ms}$ period, we double it to 0.04 time units for calculating the position. After generating code again, we could validate our assumption, which leads to a new requirement to trigger the functionality of the odometry with a period of $40 \mathrm{~ms}$. Using code generators for automatically deriving the implementation realizing the behavior of initially created models support the

${ }^{11} \overline{\text { Discretization }}$ is applied at different levels. E.g., fixed point variables are used for the implementation at the data level or time continuous differential equations are mapped to discrete execution intervals at the timing level. For further details compare [4]. 
seamless migration from the model level to the implementation level as well as allow to analyze effects arising from the implementation. Therefore, we cover the aspects IV, VI, and VII developing robotic systems at this point.

\section{System Level - SystemDesk:}

For more complex system behavior resulting from the composition of multiple individual functionalities, we use the component-based architecture provided by the AUTOSAR framework. Individual functionalities provided by the MATLAB

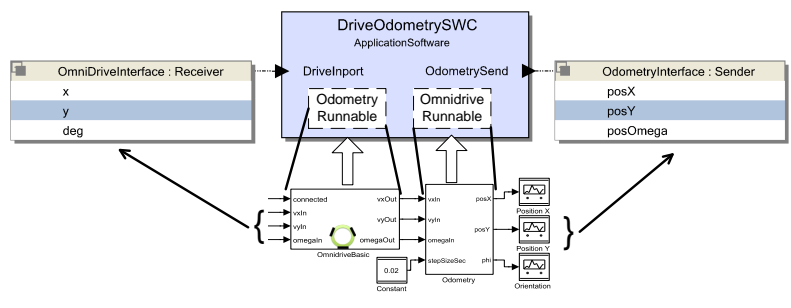

Fig. 5. Mapping from MATLAB models to SWCs. models are mapped on components such as those depicted in Fig. 5. The generated source code from TargetLink is mapped into the AUTOSAR SWC in the form of so-called Runnables.

So, the same C-Code as in the SiL simulation is used and thus, a seamless integration (VI) of individual functions into the overall system behavior is achieved. In our example, we split the MATLAB model into two Runnables, namely OdometryRunnable and OmnidriveRunnable 12 The SWC communicates to other ones over well defined ports. Furthermore, the input and output values are mapped to AUTOSAR interfaces with data entries and types respectively.

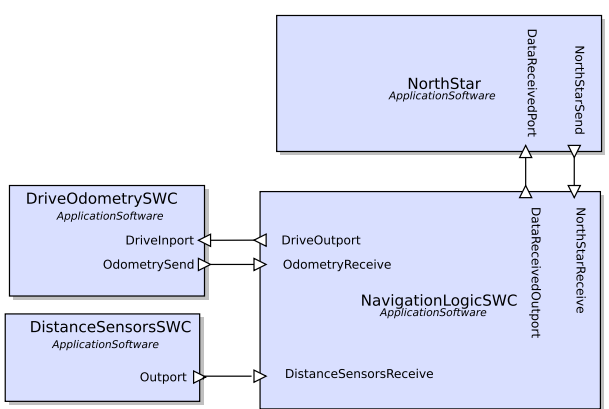

Fig. 6. SWC architecture in AUTOSAR.

The AUTOSAR architecture consists of four SWC13 (see Fig. 6). It realizes the autonomous movement of the Robotino robot and includes the SWCs DriveOdometrySWC, DistanceSensors $S W C$, NorthStar and NavigationLogicSWC. Each SWC provides the functionality such as that described previously in Section 2.1.

System Configuration: In addition to the architecture modeling and the separation of functions in different SWCs, SystemDesk supports a task specification for the underlying operating system. Runnables can be mapped to different tasks. Furthermore, several task activation events including periodic and sporadic ones

\footnotetext{
${ }^{12}$ This separation allows us to trigger the two Runnables with different periods.

${ }^{13}$ Due to a better understanding, we choose this simple excerpt of a larger architecture.
} 
are supported and additional scheduling information like periods and priorities can be modeled.

For a system simulation, one has to specify a concrete AUTOSAR conform system configuration, which includes 1) a set of tasks, each consisting of one or more Runnables, 2) one or more electronic control units, which are specialized processors, and 3) communication capabilities (buses) with a concrete mapping of messages, which have to be exchanged. In the following, we describe the first point in more detail using our running example.

The Runnables DistanceSensor, OmniDrive and CalculateDriveSpeed are mapped to an OS task, which is executed with a period of $20 \mathrm{~ms}$. A second task with the derived period of $40 \mathrm{~ms}$ contains the Runnable Odometry (cf. Section 3.2). The resulting execution of the Runnables and the schedule of the tasks is depicted in the upper time line of Fig. 7. These four basic functions run under hard realtime constraints, so we can be sure that all deadlines are met.

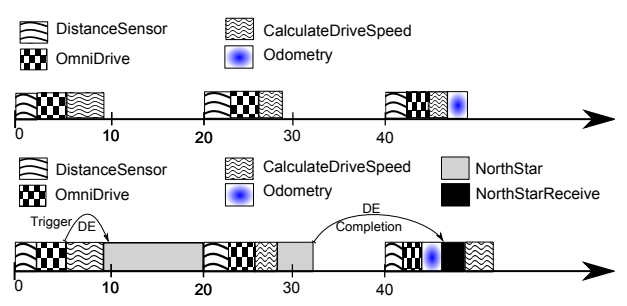

Fig. 7. Upper time line: scheduling of hard real-time functions. Lower time line: combined hard and soft real-time scheduling.

After adding more information to satisfy points 2) and 3), SystemDesk can realize a system simulation. It automatically generates the required simulation framework code according to the AUTOSAR standard, e.g., the RTE, messages, task bodies and trigger events. Furthermore, existing source files, generated by TargetLink (from the MATLAB models), are compiled and linked into the tasks. The complete system runs in a special simulation environment inside the SystemDesk tool and considers the HW configuration as well as OS task specifics. Again, this simulation is executed on a host PC and thus belongs to the prototyping stage. As depicted in Fig. 2 and the appropriate second column in Table 1, we can validate the overall system behavior in the three following scenarios considering the aspects (VI, VII, and VIII): First, we can monitor different output values, messages and variables inside the simulation environment itself. Second, we can connect the Robotino simulation environment as a plant model, which interacts with the SystemDesk tool. Finally, we are able to replace the plant simulator with the real robot. Therefore, we have to establish a WLAN connection for the communication and to access the real sensors as well as actuators. Unfortunately, this unpredictable connection can destroy the timing behavior of the simulation, although the simulator tries to keep all deadlines. If we find errors during our validation processes, we can change the configuration, architecture or communication possibilities in SystemDesk and run our simulations again. Furthermore, we are able to re-import SWCs into MATLAB and therefore, switch between the different development stages. 
According to the stage description in 4], Hardware-in-the-loop (HiL) simulations can be applied in the prototyping stage too. In these kind of simulations, the "unlimited" execution and testing hardware is often replaced by special evaluation boards with additional debugging and calibration interfaces, which are similar to the final hardware configuration. Due to limitations of our robot laboratory and missing evaluation boards, we do not use such HiL simulations. However, the integration of such boards can be carried out easily in the SystemDesk tool by changing the HW specification during the system configuration step.

Adaptation to Robotic Systems: In contrast to classic (hard) real-time applications in the domain of automotive embedded systems, robotic systems must realize functionalities, for which worst-case execution times (WCETs) are hard or impossible to predict. As a result, the integration of such behavior can only guarantee soft real-time constraints. In our application example, we use the NorthStar sensor, which is accessed via a serial USB port. Due to the fact that we use the default Linux OS driver, the timing behavior is unpredictable for that port. Additionally, we implement the navigation logic, which uses this NorthStar sensor, with library function from the MRPT library (cf. Section 2.1) for maintaining the map information of the explored topology. This includes the dynamic instantiation of an unknown number of $\mathrm{C}++$ objects (classes) at runtime, what hinders the WCET estimation, too. Therefore, the WCET can rarely be estimated at the range of a few milliseconds.

Due to te fact that AUTOSAR does not directly support such a combination of soft and hard real-time behavior, we need to adapt the framework to realize it in such a way that: (1) the schedule guarantees the preservation of hard real-time constraints for the basic functionality and (2) the communication between soft and hard real-time functionality is achieved as such that only consistent data is read.

In the first step, we separate the hard and soft real-time functions/ Runnables and map them onto different OS tasks. A soft real-time task can be configured with a lower priority in such a way that it will be interrupted by all hard realtime tasks with a higher priority. Following this development guideline achieves the first requirement (1). For the second one, we use special data events (DE) in combination with Sender/Receiver-interfaces of the AUTOSAR standard. Such events can be used to trigger the execution of Runnables inside an OS task. A DE is sent from the hard real-time task (resp. Runnable) to trigger the execution of the soft real-time Runnable. The interruptible soft real-time function produces another DE, iff, the requested output data is in a consistent state (2). The hard real-time task can read the data in its next period and triggers the soft real-time function again if required.

The lower time line in Fig. 7 illustrates the combined scheduling of soft and hard real-time tasks. The soft real-time task is triggered via a DE generated by the OmniDrive Runnable. During execution, it is preempted in order to ensure the timing deadlines of the other hard real-time Runnables. After the NorthStar Runnable has finished its execution, it sends another DE to indicate completion, 
which includes that the consistent data results can be read in the next period of the OmniDrive Runnable.

Our described development approach supports the prototyping stage of robotic systems very well. We are able to incrementally refine more and more information to specify the system while seamlessly integrating artifacts of the previous stages (VI). Activities like function development and system configuration can be applied in a round-trip engineering approach (I, II). First, we develop the control functions in MATLAB (II). Afterwards, we generate code using the TargetLink code generation capabilities (IV). At this point, we can manually integrate additional, arbitrary functionality in $\mathrm{C} / \mathrm{C}++$ or use existing libraries (V). As soon as sufficient code artifacts and libraries are provided, we are able to use the code generation and simulation capabilities of the SystemDesk tool (IV, VII). Existing SWCs, e.g., developed in a previous project can be seamlessly integrated into the system architecture and new components can be exported as library elements for other projects. Additionally, we have shown the idea of creating a combination of hard and soft real-time tasks using the AUTOSAR framework during this stage (VIII).

\subsection{Pre-Production Stage}

Within the pre-production stage, usually, a prototype of the real system is built. This prototype is tested against external environmental influences (such as temperature, vibration or other disturbances). The goal of this stage is to prove whether all requirements and constraints are still met on the real HW. During this last integration of all components and system parts, upcoming problems should be fixed as early as possible and before the final production of the product starts 4]. In our setting, we did not built any HW prototypes. Instead, we integrate the overall functions, components as well as the generated RTE and tasks to a complete system, compile and run it on the target processor of the robot ${ }^{14}$. So in this last step, we have no simulation semantic and W-LAN connection to other tools. We can fully operate the behavior of the robot in hard real-time. For verification, we use some hard real-time logging mechanism of the robot OS. Furthermore, we can change the hardware composition of the robot by adding or removing special sensors and actuators (see Section 2.1).

\section{Related Work}

Tackling the complexity of robotic and other embedded systems, we found a great deal of previous work covering partial aspects of developing such systems. According to our found aspects in Section 1 and our focus on the automotive domain, we combine the existing development methodology from [4] and the AUTOSAR standard. We evaluate our approach in a robotic production scenario using the component-based AUTOSAR architecture [12]. Other frameworks often cover only parts of the found aspects. RT-Middleware [1, 2] and ORCA [5]

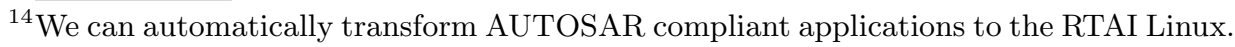


focus on the specification of components including interfaces and ports (aspects IV, V, and VII). They lack the integration of an overall methodology as well as architecture specification. Very similar to the AUTOSAR approach but with the focus on the robotic domain is the MOAST framework [3], which covers the points II, III, IV, and partially VII. However, a seamless integration of an overall methodology and the support for different tools is missing. A good comparison with other frameworks can be found in 8].

In the embedded world, testing and simulation are the major activities to verify the behavior of the system [4]. We have made intensive use of the MATLAB, Robotino and SystemDesk simulators. However, other simulators like Gazebo 7 or Webots 9] are applicable as well.

Furthermore, there are other tools for modeling and simulation of AUTOSAR conform parts of the system architecture as the Real-Time Workshop(RTW) (MATLAB extension) from the MathWorks company ${ }^{15}$ The RTW extension is limited to component functionality and interfaces. The overall system architecture description is needed beforehand 11. Parts of this description can be built by the Volcano Vehicle Systems Architect ${ }^{16}$ (VSA), which can import and export AUTOSAR conform architecture description [10. However, all these different tools can be used instead of the tools presented in this paper, if the integration in the overall methodology as well as the support for the different development stages is guaranteed.

Considering real-time constraints and combining hard and soft real-time tasks are important because of the support for library functionalities in different use-cases. For example, our navigation logic in this paper cannot be done in a predictive amount of time. Combining soft and hard real-time guarantees (1) a basic hard real-time behavior of the robotic system and (2) supports the development of complex algorithm and higher system components. Existing robotic frameworks as the Robot Operating System ${ }^{17}$ or Microsoft Robotics Studid ${ }^{18}$ are well established for developing complex robotic systems. They have drawbacks concerning the integration of hart real-time constraints.

\section{Conclusion}

We have shown in this paper an overall methodology (I) along with different exemplary development activities as well as artifacts on different levels of abstraction (II, III). We know that not all tasks can be executed in HRT. Therefore, we have shown the idea of combining different hard and soft real-time tasks into the overall system using the AUTOSAR approach (VIII). Furthermore, we are able to integrate several tools and external libraries into our overall toolchain (IV, V, VI). However, we are not limited to the tools we show in this paper. This provided flexibility is stabilized by a clear structure of different development stages

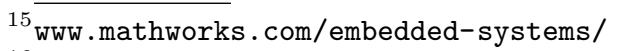

16 WWW.mentor.com/

17 wWw.ros.org

18 WWW.microsoft.com/robotics/
} 
(III) allowing a round-trip engineering for different functions, the integration of components as well as the simulation and testing of the development artifacts to the point of the complete system on the target platform. Therefore, we adapt ideas of the automotive domain to the development of robotic systems.

As future work, we want to build a complex robot production scenario applying the proposed methodology of this paper and evaluate the interaction of soft and hard real-time system parts.

\section{References}

1. Ando, N., Suehiro, T., Kitagaki, K., Kotoku, T., Woo-Keun, Y.: RT-middleware: distributed component middleware for RT (robot technology). In: 2005 IEEE/RSJ International Conference on Intelligent Robots and Systems. pp. 3933 - 3938 (2005)

2. Ando, N., Suehiro, T., Kotoku, T.: A software platform for component based rtsystem development: Openrtm-aist. In: Proceedings of the 1st International Conference on Simulation, Modeling, and Programming for Autonomous Robots. pp. 87-98. SIMPAR '08, Springer, Berlin, Heidelberg (2008)

3. Balakirsky, S., Proctor, F.M., Scrapper, C.J., Kramer, T.R.: A mobile robot control framework: From simulation to reality. In: Proceedings of the 1st International Conference on Simulation, Modeling, and Programming for Autonomous Robots. pp. 111-122. SIMPAR '08, Springer, Berlin, Heidelberg (2008)

4. Broekman, B., Notenboom, E.: Testing Embedded Software. Wesley (2003)

5. Brooks, A., Kaupp, T., Makarenko, A., Williams, S., Oreback, A.: Towards component-based robotics. In: International Conference on Intelligent Robots and Systems. pp. $163-168$ (2005)

6. Giese, H., Neumann, S., Niggemann, O., Schätz, B.: Model-Based Integration. In: Model-Based Engineering of Embedded Real-Time Systems, Dagstuhl Castle, Germany. LNCS, vol. 6100, pp. 17-54. Springer (2011)

7. Koenig, N., Howard, A.: Design and use paradigms for gazebo, an open-source multi-robot simulator. In: In IEEE/RSJ International Conference on Intelligent Robots and Systems. pp. 2149-2154 (2004)

8. Manso, L., Bachiller, P., Bustos, P., Núñez, P., Cintas, R., Calderita, L.: Robocomp: a tool-based robotics framework. In: Proceedings of the 2nd international conference on Simulation, modeling, and programming for autonomous robots. pp. 251-262. SIMPAR'10, Springer, Berlin, Heidelberg (2010)

9. Michel, O.: Webots: Professional Mobile Robot Simulation. International Journal of Advanced Robotic Systems 1, 39-42 (2004)

10. Sandmann, G., Seibt, M.: AUTOSAR-Compliant Development Workflows: From Architecture to Implementation - Tool Interoperability for Round-Trip Engineering and Verification and Validation. Tech. Rep. 2012-01-0962, SAE International (2012)

11. Sandmann, G., Thompson, R.: Development of AUTOSAR Software Components within Model-Based Design. Tech. Rep. 2008-01-0383, SAE International (2008)

12. http://www.autosar.org/ AUTOSAR_EXP_LayeredSoftwareArchitecture.pdf (2011), page id: 94 ju5 